\title{
Evaluation of Cover and Reflector in Receiver Antennas for SM-MIMO Wireless Communications
}

\author{
Shingo Yoshizawa ${ }^{1}$ and Yoshikazu Miyanaga ${ }^{2}$ \\ ${ }^{1}$ Department of Electrical and Electronic Engineering, Kitami Institute of Technology, 165, Koen-cho, Kitami, \\ Hokkaido 090-8507, Japan \\ ${ }^{2}$ Graduate School of Information Science and Technology, Hokkaido University, Kita-14 Nishi-9, Kita-ku, Sapporo, \\ Hokkaido 060-0814, Japan \\ Correspondence should be addressed to Shingo Yoshizawa; yosizawa@mail.kitami-it.ac.jp
}

Received 22 August 2013; Revised 28 October 2013; Accepted 1 November 2013

Academic Editor: George Tsoulos

Copyright (C) 2013 S. Yoshizawa and Y. Miyanaga. This is an open access article distributed under the Creative Commons Attribution License, which permits unrestricted use, distribution, and reproduction in any medium, provided the original work is properly cited.

\begin{abstract}
Spatial multiplexing multiple-input multiple-output (SM-MIMO) is effective in increasing communication throughput. However, performance degradation occurs in poor multipath and line-of-sight (LOS) channels due to the high spatial correlation. This paper presents the evaluation of cover and reflector in receiver antennas to overcome the performance degradation in MIMO LOS channels. We measured the characteristics of packet error rate (PER), signal-to-noise ratio (SNR), spatial correlation coefficient, and received power ratio in farm, groove, passage, and corner environments. The farm environment degraded communication performance due to the high spatial correlation coefficient. Following the measurement results, we fabricated cover and reflector in receiver antennas to decrease the high spatial correlation and confirmed the improvement of communication performance.
\end{abstract}

\section{Introduction}

A multiple-input multiple-output (MIMO) [1] technique can improve communication reliability or increase data throughput and attracts a great deal of attention in current wireless communications. Spatial multiplexing (SM) enables independent and parallel data transmission in spatial domain; however, its communication performance strongly depends on MIMO channel conditions. For poor multipath and line-of-sight (LOS) conditions, the high spatial correlation induces performance degradation. In our previous work, we have reported outdoor evaluation of $2 \times 2$ MIMO-OFDM communication in farm and passage (between buildings) environments $[2,3]$. The farm condition showed the worst communication performance due to the high spatial correlation.

To overcome the performance degradation of SM-MIMO, antenna polarization [4] and transmit-array that lies between transmitter and receiver $[5,6]$ and simulation analysis of near-field MIMO communication with back reflector [7] have been presented. However, antenna polarization is affected by cross-polarization discrimination depending on propagation environments. Their transmission characteristics might be degraded by weather or obstacles. The transmitarray restricts the locations because the transmit-array should be placed away from the transmit and receive antennas. Our approach is use of cover and reflector in MIMO receiver antennas, which does not rely on polarization and transmitarray and is suitable for long-distance communication. First, we evaluated communication characteristics of farm, groove, passage, and corner environments corresponding to rich/poor multipath and LOS/non-LOS (NLOS) conditions. The parameters of signal-to-noise ratio (SNR), spatial correlation coefficient, and received power ratio were important to prospect communication performance. Following the measurements, we fabricated cover and reflector to coordinate the parameters of spatial correlation coefficient and received power ratio. By using the cover and reflector, we confirmed the improvement of communication performance in the farm environment.

The paper is organized as follows. Section 2 reports the outdoor experiment in basic antenna. Section 3 describes 


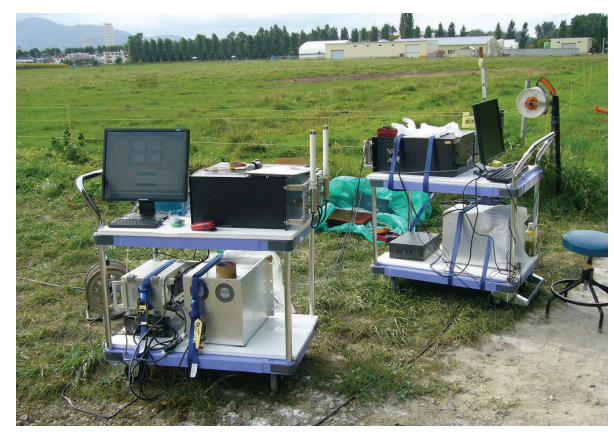

FIGURE 1: Photograph of experimental platform.

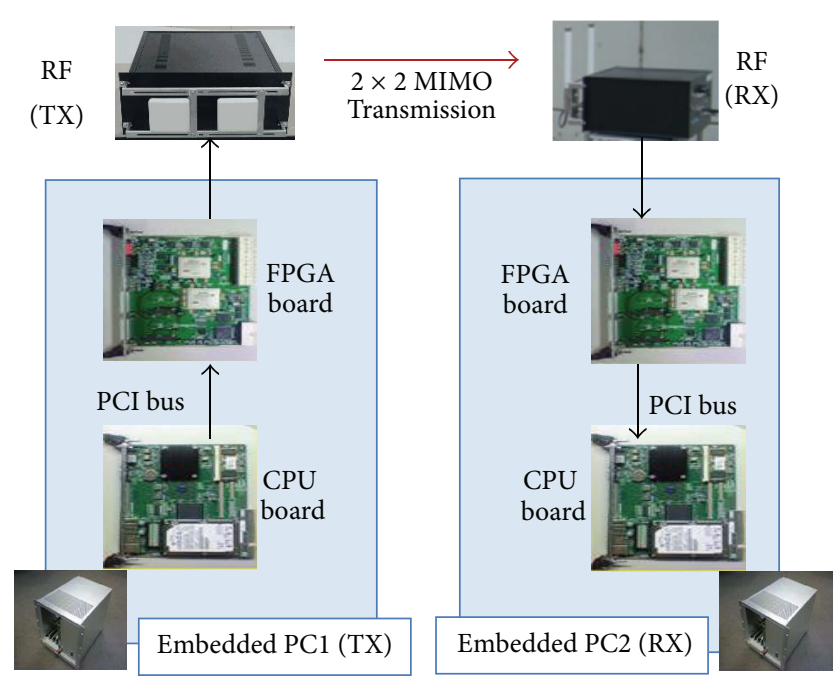

Figure 2: Structure of experimental platform for $2 \times 2$ MIMOOFDM communication.

the fabrication of cover and reflector in MIMO receiver antennas. The evaluation of the proposed cover and reflector is presented in Section 4. Section 5 summarizes the paper.

\section{Outdoor Experiment for Basic Antenna}

2.1. Experimental Platform. The experimental platform that was developed in our previous work [2] is depicted in Figure 1. The platform structure for $2 \times 2$ MIMO-OFDM communication is illustrated in Figure 2. Open-loop spatial multiplexing (OLSM) that does not share channel state information (CSI) in transmitter and receiver is adopted in the MIMO-OFDM communication. The platform consists of the baseband units with the CPU and FPGA boards and the RF units. The CPU board generates transmit data, records received data on software processing, and evaluates the packet error rate (PER), signal-to-noise ratio (SNR), and MIMO propagation channel. The FPGA board provides MIMO-OFDM modulation/demodulation on hardware processing. The baseband signal bandwidth of OFDM is about $80 \mathrm{MHz}$ according to the similar specifications of IEEE 802.1lac WLAN [8]. The RF unit is designed by superheterodyne architecture modulating 374 and 5,200 MHz in IF and
TABLE 1: Experimental conditions.

\begin{tabular}{ll}
\hline RF band & $5150-5250 \mathrm{MHz}$ \\
Signal bandwidth & $79.68 \mathrm{MHz}$ \\
Number of subcarriers & 512 \\
Max. trans. power & $357 \mathrm{~mW}$ \\
& NATEC PAT509S-4953 \\
TX antenna (directional) & Gain 9 dBi \\
& Half-power angle \\
& E-plane 58 deg/H-plane 76 deg \\
RX antenna & SANSEI ELECTRIC ANTDP-010A0 \\
(omnidirectional) & Gain 0 dBi \\
& E-plane 360 deg/H-plane 360 deg \\
Antenna height & $1 \mathrm{~m}$ \\
Distance (TX-RX) & $40 \mathrm{~m}$ \\
MIMO detection & MMSE \\
Coding rate & $1 / 2$ \\
Modulation & 16 QAM \\
\hline
\end{tabular}

RF bands, respectively. Figure 3 shows a block diagram of $2 \times 2$ MIMO-OFDM transmitter and receiver. The functions in the transmitter and receiver blocks are the same as those of IEEE802.11n PHY. The MIMO detection block recovers data symbols from spatially multiplexed data streams for SMMIMO. The linear detection based on a minimum meansquare-error (MMSE) criterion is adopted in the MIMO detection block.

2.2. Experimental Conditions. Table 1 shows the experimental conditions for $2 \times 2 \mathrm{MIMO}-\mathrm{OFDM}$ communication. The transmitter antenna has a directional characteristic where the half-power angles are 58 and 76 degrees in E- and H-planes, respectively. The receiver antenna has an omnidirectional characteristic with 360 degrees in both E- and H-planes. The experimental place is illustrated in Figure 4. We evaluate communication characteristics in passage (between buildings), corner (of building), farm, and groove environments. Their photographs are shown in Figure 5. The passage and corner environments represent rich multipath conditions due to the many reflected waves from buildings. The farm and groove environments show poor multipath conditions. The LOS conditions can be observed in the passage and farm environments. The NLOS conditions correspond to the corner and groove environments. The antenna location of the transmitter and receiver antennas common to every environment is denoted in Figure 6. The distance between transmitter and receiver is $40 \mathrm{~m}$. The height of both antennas is $1 \mathrm{~m}$. The offset of $0.8 \mathrm{~m}$ at the $y$-axis corresponds to the location errors in actual wireless systems, whose influence is discussed in Section 3.

The packet format is shown in Figure 7. The packet consists of short training field (STF) for packet detection, long training field (LTF) for measured SNR and MIMO channel estimation, and data symbols (Data). The measured SNR is computed from the long training field. The OFDM symbol duration in "LTF" and "Data" is $7.2 \mu$ s with the FFT/IFFT window length of $6.4 \mu \mathrm{s}$ and the guard interval of $0.8 \mu \mathrm{s}$. 

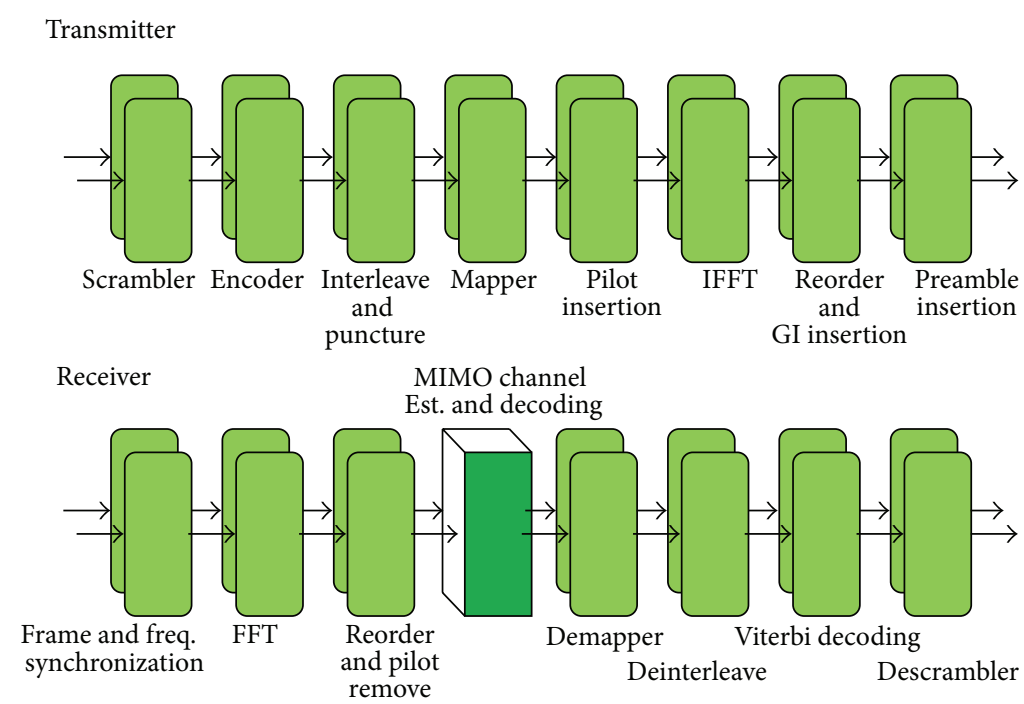

FIGURE 3: Block diagram of $2 \times 2$ MIMO-OFDM transmitter and receiver.

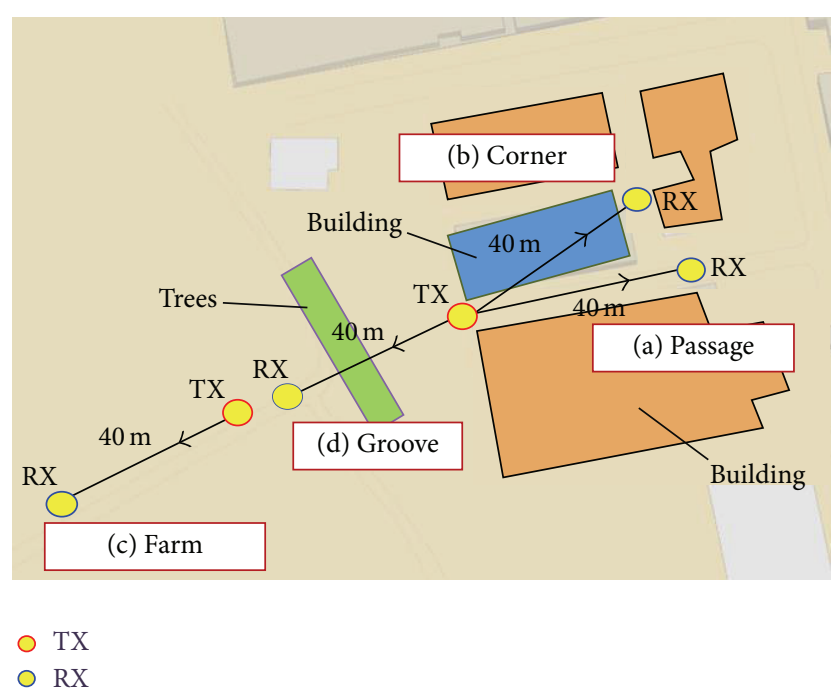

FIGURE 4: Experimental place for passage, corner, farm, and groove conditions in Hokkaido University Sapporo campus.

The number of OFDM symbols in 16-QAM transmission is 10. If the number of OFDM symbols is infinity, the ideal data rate reaches $266 \mathrm{Mbps}$. The PER is measured by counting the number of packet errors in the receiver, where the same binary data in data encoding is known in both the transmitter and the receiver.

Here, we remark the parameters of spatial correlation coefficient and received power ratio used in the evaluation of experimental results. Given by a MIMO channel matrix $\mathbf{H}$, its matrix elements are expressed as

$$
\mathbf{H}=\left(\begin{array}{ll}
h_{11} & h_{12} \\
h_{21} & h_{22}
\end{array}\right) .
$$

For $M_{T}$ transmitter antennas and $M_{R}$ receiver antennas, the MIMO channel $\mathbf{H}$ is represented by the $M_{R} \times M_{T}$ matrix.
Given a $2 \times 2$ MIMO channel matrix, the spatial correlation coefficient is given by the following equation [9]:

$$
\gamma=\frac{h_{11} h_{21}^{*}+h_{12} h_{22}^{*}}{\sqrt{\left(\left|h_{11}\right|^{2}+\left|h_{12}\right|^{2}\right)\left(\left|h_{21}\right|^{2}+\left|h_{22}\right|^{2}\right)}},
$$

where $\{\cdot\}^{*}$ denotes complex symmetry. The received power ratio providing the difference of powers between two received antennas is given by

$$
\beta=\frac{\left|h_{11}\right|^{2}+\left|h_{12}\right|^{2}}{\left|h_{11}\right|^{2}+\left|h_{12}\right|^{2}+\left|h_{21}\right|^{2}+\left|h_{22}\right|^{2}} .
$$

The value of 0.5 indicates that the power values of two receiver antennas are equal. Since the MIMO channel capacity [1] is formulated by

$$
C=\log _{2} \operatorname{det}\left(\mathbf{I}+\frac{\rho}{N_{t}} \mathbf{H} \mathbf{H}^{H}\right),
$$

the MIMO channel capacity can be expressed by the spatial correlation coefficient and received power ratio [9] as

$$
C=\log _{2}\left(1+\rho+\beta \rho^{2}(1-\beta)\left(1-|\gamma|^{2}\right)\right),
$$

where $\rho$ is the defined as $\rho=P / N_{0}$. $P$ is the transmit power and $N_{0}$ is the noise power. I is an identity matrix. $N_{t}$ denotes the number of transmitter antennas. The influence of high spatial correlation in terms of channel capacity by $\log _{2}(1-$ $\left.|\gamma|^{2}\right)$ is extracted from (5). The relation between spatial correlation coefficient and channel capacity is illustrated in Figure 8. When the threshold value is set to 0.99 for horizontal axis denoted by the broken line, channel capacity exponentially decreases in the region of $\gamma \geq 0.99$. The influence of received power ratio by the term of $\log _{2} \beta(1-\beta)$ appears when the received power ratio considerably goes away from 0.5. 


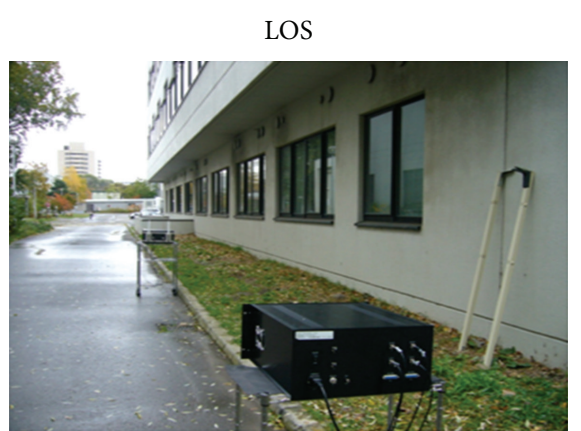

(a) Passage

LOS

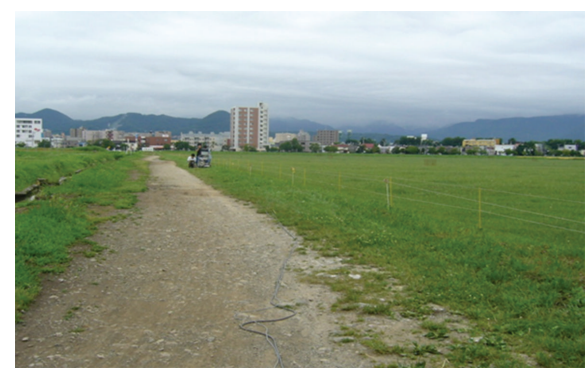

(c) Farm

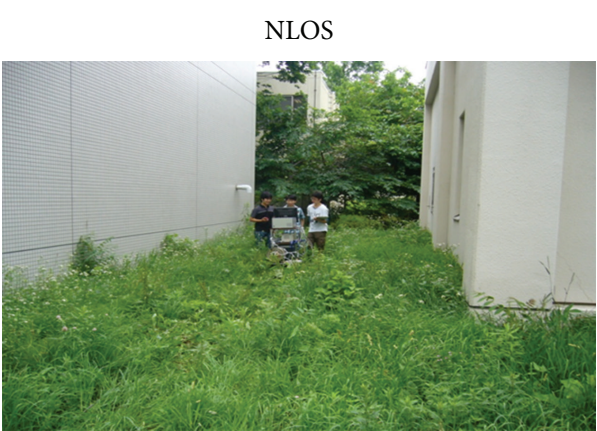

(b) Corner

NLOS

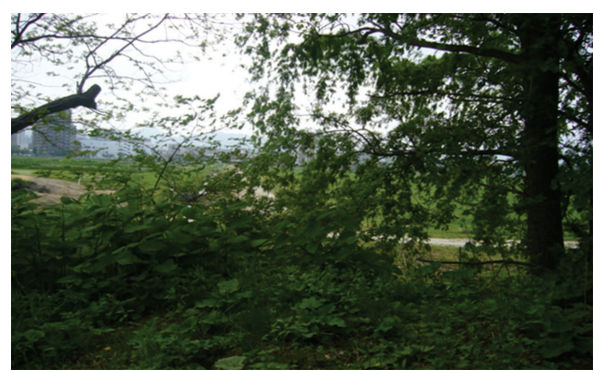

(d) Groove

FIGURE 5: Photographs of experimental environments.

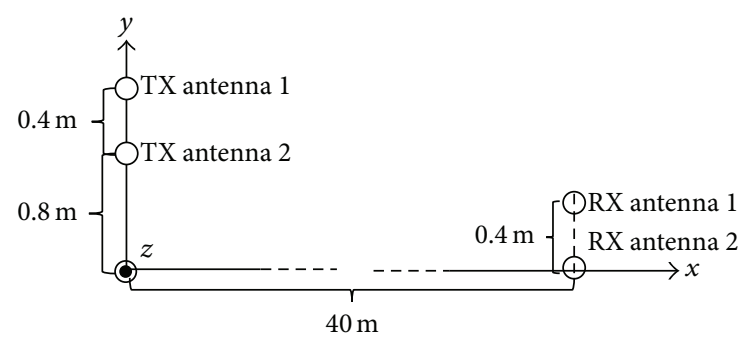

FIGURE 6: Antenna location of transmitter and receiver antennas.

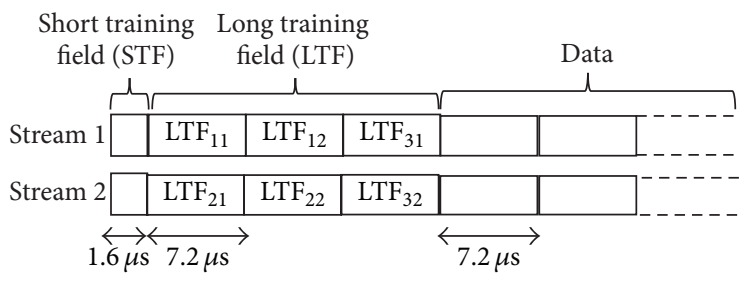

Figure 7: Frame format.

Due to the complicated influences of RF demodulator and analog-to-digital converter, we cannot know the SNR directly in the experimental platform. We use the measured SNR estimated from the training symbols in place of the SNR. In the $2 \times 2$ MIMO-OFDM system, the frequency-domain signal is expressed as

$$
\mathbf{y}(l)=\mathbf{H s}(l)+\mathbf{n}(l),
$$

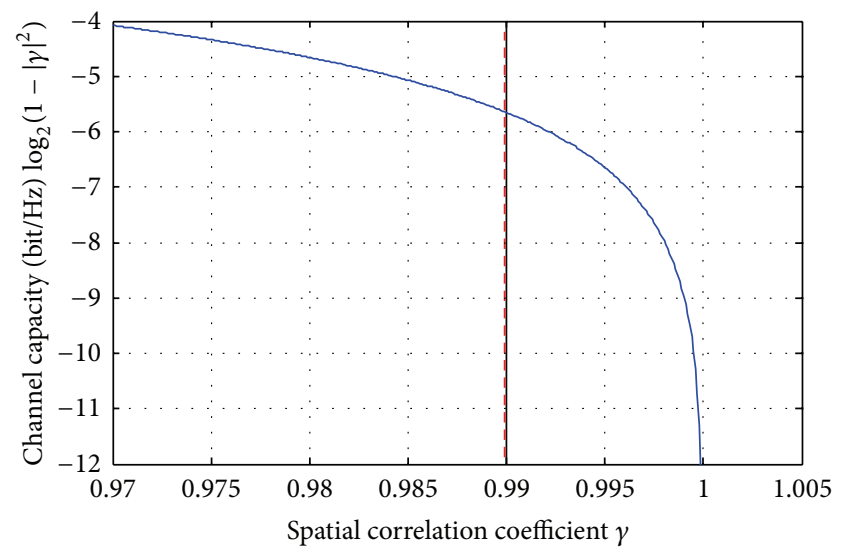

FIGURE 8: Relation between spatial correlation coefficient and channel capacity.

where $\mathbf{s}(l)$ is a transmit signal with $l$ th OFDM symbol and $\mathbf{n}(l)$ is a noise signal. When the transmit signal in the first OFDM symbol (LTF 11 and $\mathrm{LTF}_{21}$ in Figure 7) is the same as in the second OFDM symbol ( $\mathrm{LTF}_{12}$ and $\mathrm{LTF}_{21}$ ),

$$
\begin{aligned}
& \mathbf{y}(1)=\mathbf{H s}(1)+\mathbf{n}(1), \\
& \mathbf{y}(2)=\mathbf{H s}(1)+\mathbf{n}(2) .
\end{aligned}
$$

The signal power $P_{e}$ is computed from the average power of $\mathbf{y}(1)$ and $\mathbf{y}(2)$ after the channel equalization. The noise power $N_{e}$ is estimated from the subtraction of $\mathbf{y}(1)$ and $\mathbf{y}(2)$. The measured SNR is given by the ratio of $P_{e}$ and $N_{e}$. 


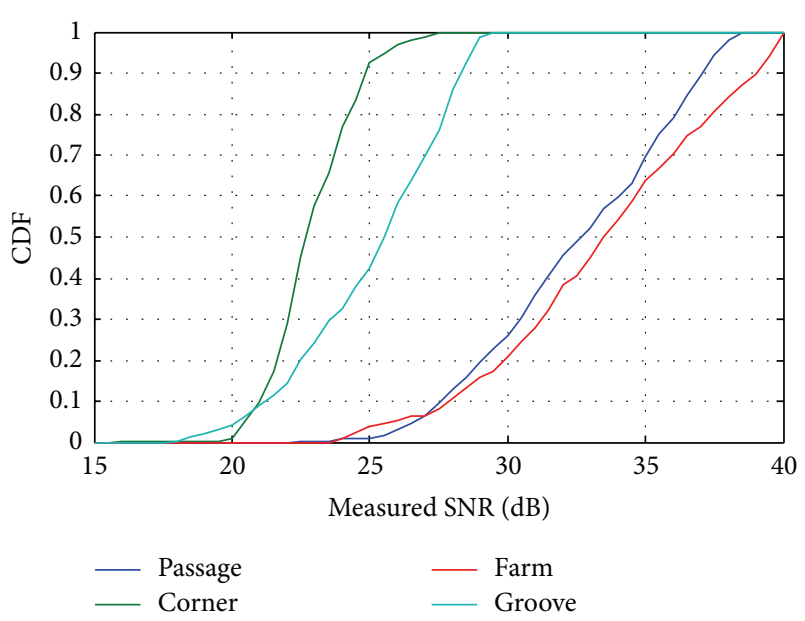

FIGURE 9: Distribution plot for measured SNR.

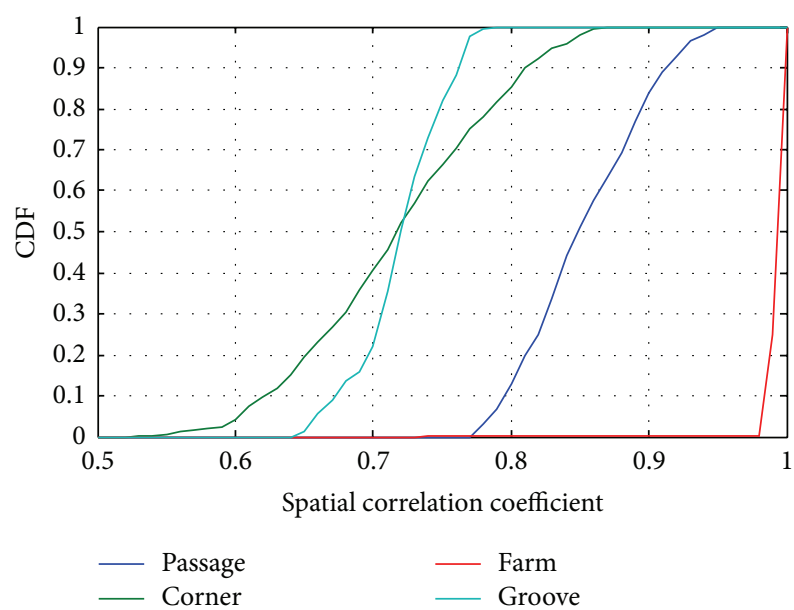

FIgURE 10: Distribution plot for spatial correlation coefficient.

2.3. Experimental Results. We compare communication characteristics of passage, corner, farm, and groove environments for "basic" (without cover and reflector) antenna. Table 2 summarizes the experimental results. The averages of PER, measured SNR, spatial correlation coefficient, received power ratio, and measured channel capacity are evaluated from 300 packets for every environment. The PER performance is ranked in the order of passage, corner, groove, and farm environments. In the measured SNR, the passage and the farm environments show high SNRs due to the LOS conditions. However, the PER performance in the farm environment is the worst due to the high spatial correlation coefficient of 0.994 .

We evaluate the distribution plots of cumulative distribution function (CDF) for measured SNR, spatial correlation coefficient, received power ratio, and measured channel capacity shown in Figures 9, 10, 11, and 12. In Figure 9, the difference of measured SNR results is also observed by the CDF. For the CDF of 0.5 , the corner and groove environments range from 22 to $26 \mathrm{~dB}$ and the passage and farm environments range from 32 to $34 \mathrm{~dB}$. In Figure 10,

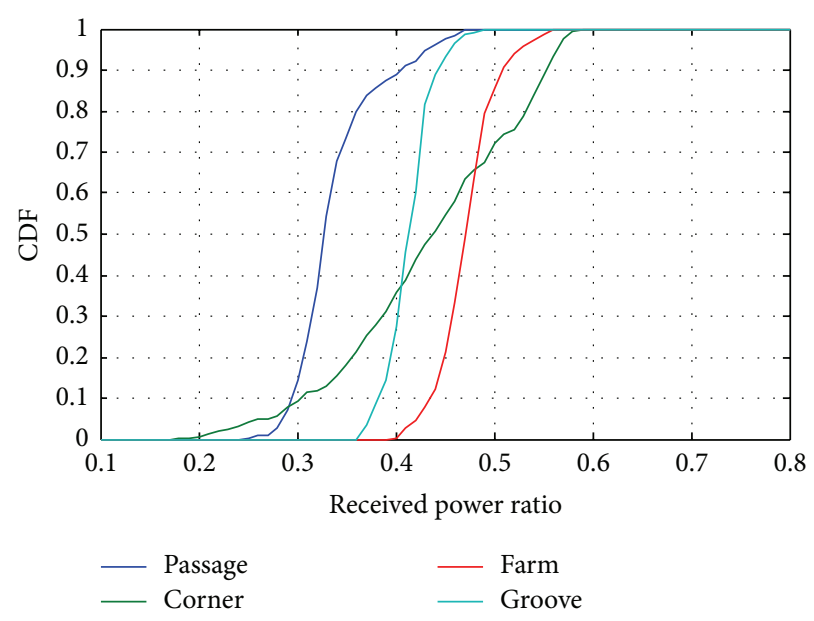

FIGURE 11: Distribution plot for received power ratio.

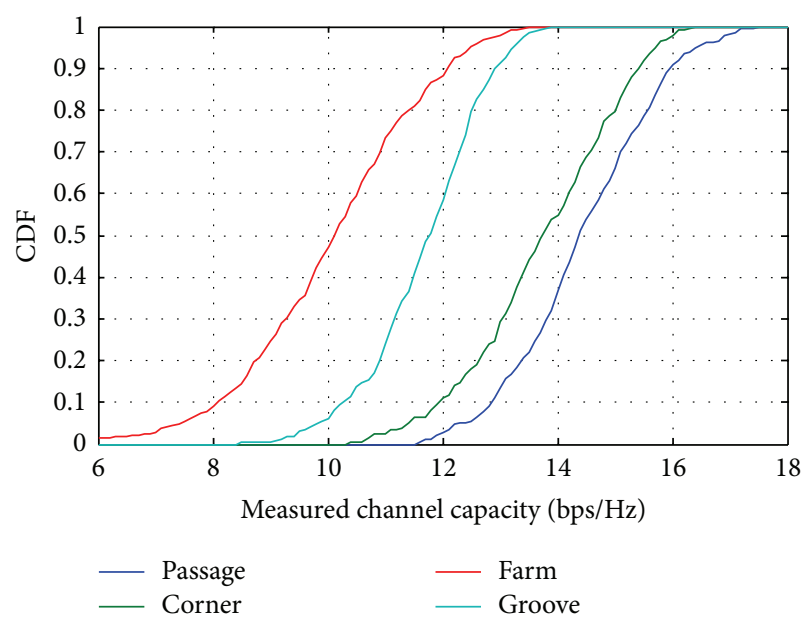

FIGURE 12: Distribution plot for measured channel capacity.

the farm environment has much higher value of the spatial correlation coefficient than the other environments.

The passage environment ranges from 0.8 to 0.9 in the spatial correlation coefficient; however, it gives a much better PER performance than the other environments. The influence of performance degradation by spatial correlation is small as long as the spatial correlation coefficient does not exceed the threshold value. This fact indicates that the farm environment has the possibility of dissolving the performance degradation if the spatial correlation coefficient can be slightly decreased. In Figure 11, the received power ratio ranges from 0.3 to 0.5 for all the environments. The results of received power ratio would not affect PER performance so much.

The measured channel capacity results are plotted in Figure 12. The measured channel capacity is computed from (4) by using the MIMO channel matrix and the measured SNR. The MIMO channel matrix is extracted from the long training field in Figure 7. Since a theoretical channel capacity gives an upper bound on the information rate for reliable communication, it does not directly correspond to the communication performance in the experimental 
TABLE 2: Summary of experimental results for basic antenna.

\begin{tabular}{lcccc}
\hline & Passage & Corner & Farm & Groove \\
\hline PER & 0.003 & 0.595 & 0.997 & 0.655 \\
Measured SNR (dB) & 32.74 & 23.12 & 33.68 & 25.33 \\
Spatial correlation coefficient & 0.857 & 0.722 & 0.994 & 0.724 \\
Received power ratio & 0.342 & 0.439 & 0.476 & 0.418 \\
Measured channel capacity (bps/Hz) & 14.48 & 13.78 & 9.96 & 11.72 \\
\hline
\end{tabular}
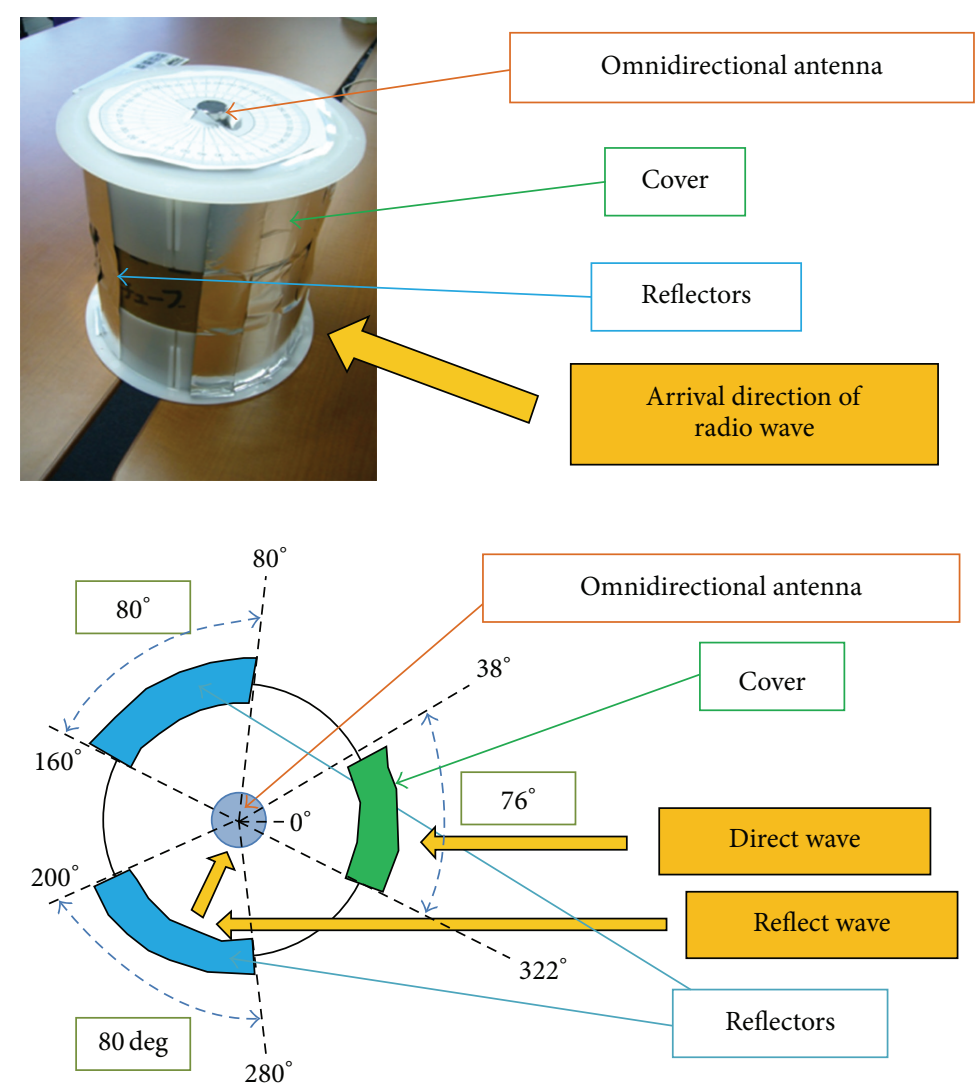

FIGURE 13: Proposed cover and reflector.

platform. However, the measured channel capacity is also ranked in the order of passage, corner, groove, and farm environments, which is the same as the PER performance. The measured channel capacity can be used as the way of the rough comparison of communication environments.

\section{Fabrication of Cover and Reflector}

We have fabricated cover and reflector attaching to MIMO receiver antennas so as to decrease the spatial correlation coefficient in the farm environment. The photograph and structure of the proposed cover and reflector are illustrated in Figure 13. The cover and reflector consist of the H-plane omnidirectional antenna and the cylindrical plastic case with the radius of $12 \mathrm{~cm}$, where the side of the case is pasted with aluminum foil. When the front cover is set to 0 degrees, the cover locates at -38 to 38 degrees and the two reflectors locate at 80 to 160 degrees and 200 to 280 degrees.
Figure 14 shows the incident paths of radio waves by the cover and reflector. Due to the offset at the $y$-axis in Figure 6, the four incident paths to the receiver antennas are not symmetric. As for basic antenna, the influence of nonsymmetric paths can be ignored because their path lengths are almost the same. When the covers are located in front of the receiver antennas in Figure 14(a), three direct waves convert to the diffracted waves. However, one direct wave incidents to the second receiver antenna owing to the nonsymmetric deployment. It gives the difference of wave types between first and second receiver antennas and contributes to decreasing the spatial correlation coefficient in the farm environment. However, the diffracted wave causes the decrease of power in the receiver antenna. It causes power unbalance for the two receiver antennas where received power ratio considerably goes away from 0.5. We apply antenna side reflectors to dissolve the power imbalance, illustrated in Figure 14(b). The side reflectors can increase the power of the first receiver 


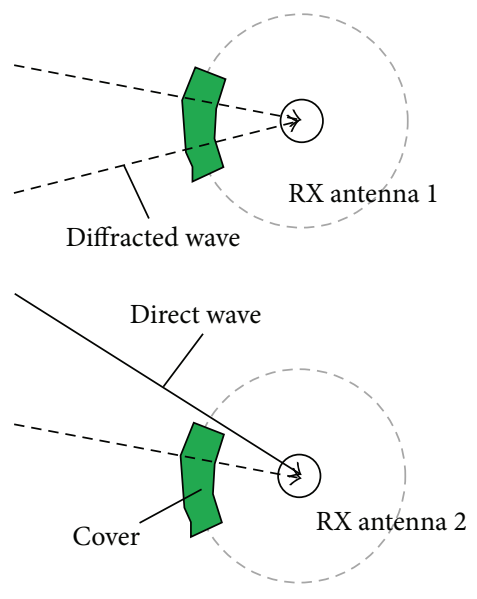

(a) Cover

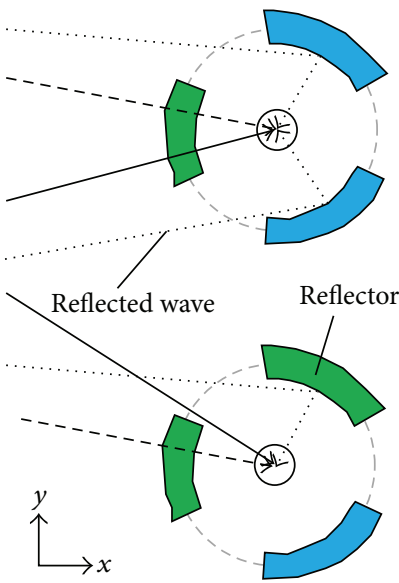

(b) Cover and reflector

FIGURE 14: Incident paths of radio waves for cover and reflector.

TABLE 3: Summary of experimental results in conventional and proposed antennas.

\begin{tabular}{lcccc}
\hline & Basic antenna & Cover & Reflector & Cover and reflector \\
\hline PER & 0.963 & 0.690 & 0.960 & 0.020 \\
Measured SNR $(\mathrm{dB})$ & 33.43 & 33.15 & 33.71 & 33.37 \\
Spatial correlation coefficient & 0.985 & 0.923 & 0.987 & 0.974 \\
Received power ratio & 0.511 & 0.181 & 0.620 & 0.283 \\
\hline
\end{tabular}

antenna by adding the reflected waves. The reflector is used for the adjustment of the received power ratio affected by the cover.

\section{Evaluation of Cover and Reflector}

We compare four types of basic antenna, cover, reflector, and "cover and reflector" in the farm environment. The experimental condition is the same as in Section 2. Table 3 summarizes the evaluation results. The averages of PER, measured SNR, spatial correlation coefficient, and received power ratio are evaluated for every 300 packets. The PER performance is ranked in the order of cover and reflector, cover, basic antenna, and reflector. In the measured SNR, the four types show similar SNR values due to the same farm environment. Although the spatial correlation coefficient of cover and reflector is slightly smaller than those of basic antenna and reflector, the considerable improvement of PER performance is observed. The first column in Table 3 has the same condition of the third column in Table 2, that is, the farm environment and the basic antenna. Due to the existence of the plastic case (without the aluminum foils) in Figure 13, the results in Table 3 are slightly different from those in Table 2.

We evaluate the distribution plots of CDF for spatial correlation coefficient and received power ratio, shown in Figures 15 and 16. In Figure 15, the decrease of the spatial correlation coefficient is observed from the results of the cover and cover and reflector. The reflector does affect spatial correlation so much. In Figure 16, the received power ratio in

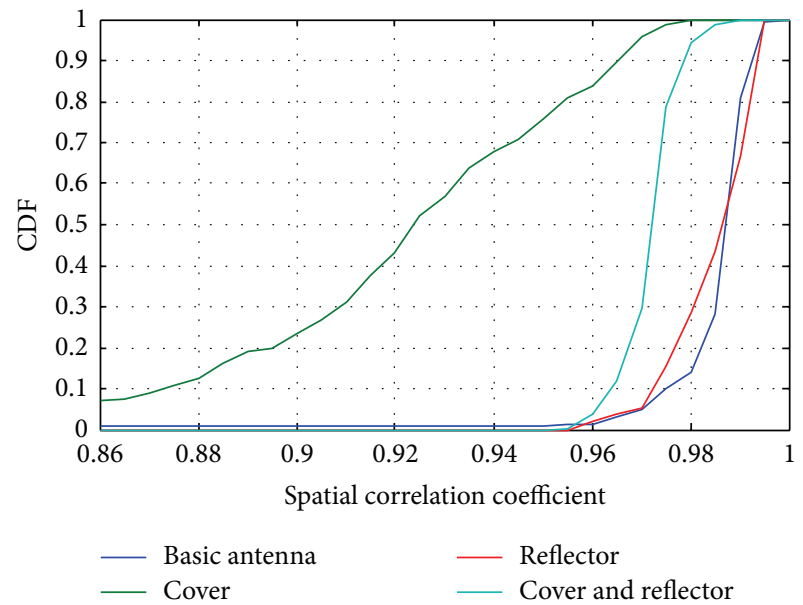

FIGURE 15: Scatter plot for spatial correlation coefficient in four types of antenna.

the cover is 0.18 for the CDF of 0.5 . This power imbalance in receiver antennas causes the performance degradation. The reflector and cover and reflector increase the received power ratio, locating at 0.61 and 0.28 for the CDF of 0.5 . It indicates the power increase of the second receiver antenna by accepting the reflected waves. The cover and reflector has a better tradeoff between spatial correlation coefficient and received power ratio and can improve PER performance compared to the basic antenna. 


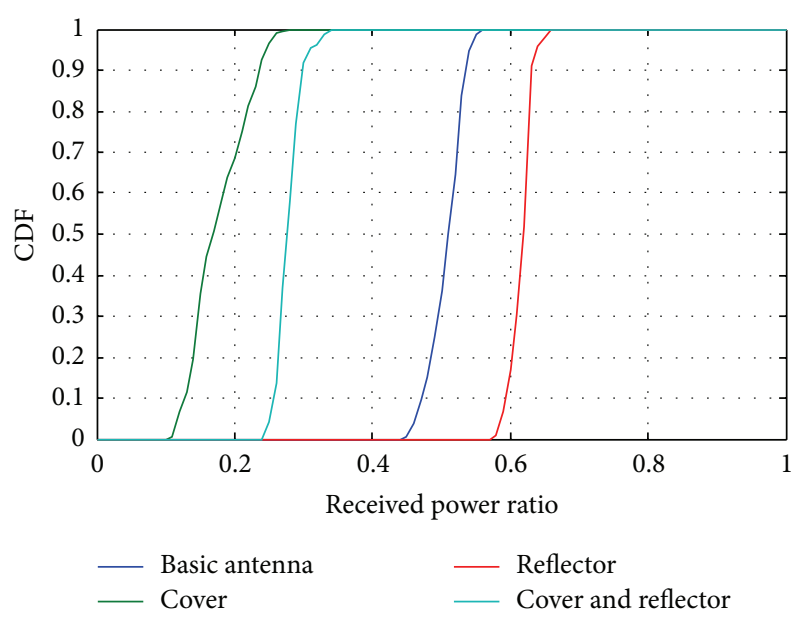

FIGURE 16: Scatter plot for received power ratio in four types of antenna.

\section{Conclusion}

This paper presents the evaluation of cover and reflector in receiver antennas so as to overcome performance degradation in MIMO LOS channels. The proposed cover and reflector slightly decreases the spatial correlation coefficient and keeps the received power ratio near 0.5. Owing to these effects, the MIMO-OFDM transceiver has attained better communication performance even in the farm environment. Evaluation of larger MIMO systems such as $4 \times 4$ MIMO will be studied as our future work.

\section{Conflict of Interests}

The authors declare that there is no conflict of interests regarding the publication of this paper.

\section{References}

[1] G. J. Foschini and M. J. Gans, "On limits of wireless communications in a fading environment when using multiple antennas," Wireless Personal Communications, vol. 6, no. 3, pp. 311-335, 1998.

[2] S. Yoshizawa, S. Odagiri, Y. Asai, T. Gunji, T. Saito, and Y. Miyanaga, "Development and outdoor evaluation of an experimental platform in an $80-\mathrm{MHz}$ bandwidth $2 \times 2$ MIMOOFDM system at 5.2-GHz band," in Proceedings of the 21st IEEE International Symposium on Personal Indoor and Mobile Radio Communications (PIMRC '10), pp. 1049-1054, September 2010.

[3] S. Yoshizawa, M. Sugitani, and Y. Miyanaga, "Effectiveness of cover and reflector in receiver antennas for MIMO line-ofsight channels," in Proceedings of the International Symposium on Personal, Indoor and Mobile Radio Communications (PIMRC '13), September 2013.

[4] P. Kyritsi, D. C. Cox, R. A. Valenzuela, and P. W. Wolniansky, "Effect of antenna polarization on the capacity of a multiple element system in an indoor environment," IEEE Journal on Selected Areas in Communications, vol. 20, no. 6, pp. 1227-1239, 2002.
[5] J. Shen, Y. Oda, T. Furuno, T. Maruyama, and T. Ohya, "A novel approach for capacity improvement of $2 \times 2$ MIMO in LOS channel using reflectarray," in Proceedings of the 73rd IEEE Vehicular Technology Conference (VTC-Spring '11), pp. 1-5, May 2011.

[6] Y. Takahashi, N. Honma, and Y. Suzuki, "Improvement of MIMO channel cpacity using tunable transmit-array antenna," in Proceedings of the International Symposium on Antennas and Propagation (ISAP '12), pp. 283-286, October 2012.

[7] H. Hirayama, G. Matsui, N. Kikuma, and K. Sakakibara, "Improvement of channel capacity of near-field MIMO," in Proceedings of the 4th European Conference on Antennas and Propagation (EuCAP '10), pp. 1-4, April 2010.

[8] "Wireless LAN Medium Access Control (MAC) and Physical Layer (PHY) Specifications: Enhancements for Very High Throughput for Operation in Bands below 6GHz," IEEE P802.11ac/ D1.0 Std., January 2011.

[9] S. L. Loyka, "Channel capacity of two-antenna BLAST architecture," Electronics Letters, vol. 35, no. 17, pp. 1421-1422, 1999. 

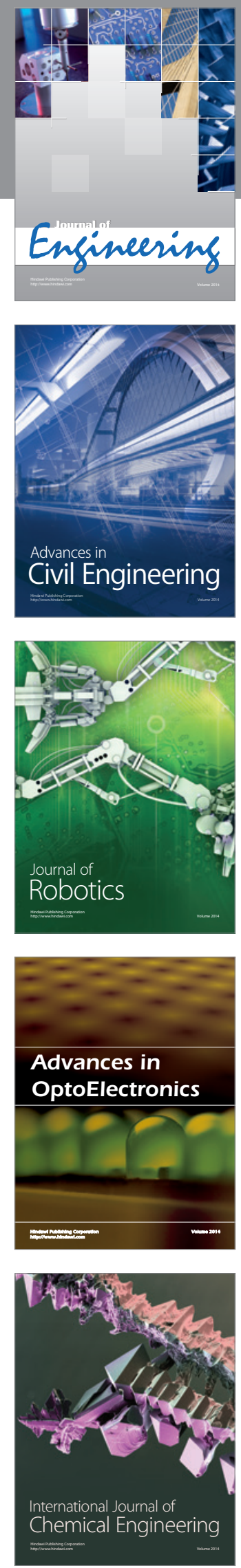

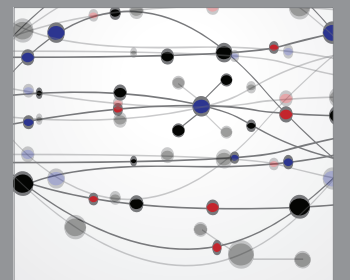

The Scientific World Journal
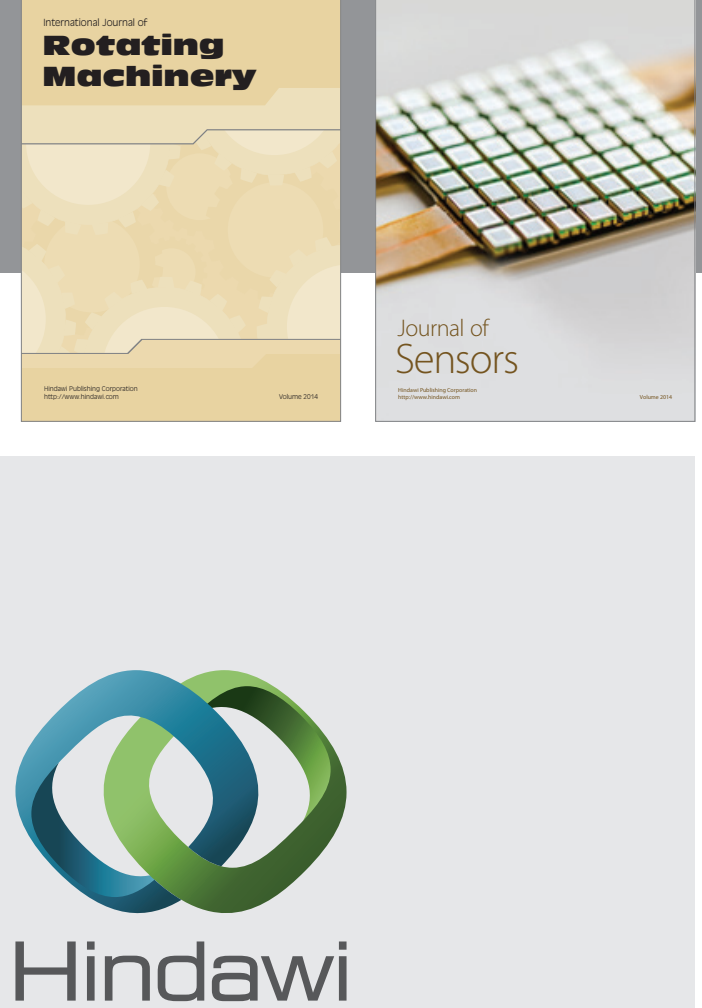

Submit your manuscripts at http://www.hindawi.com
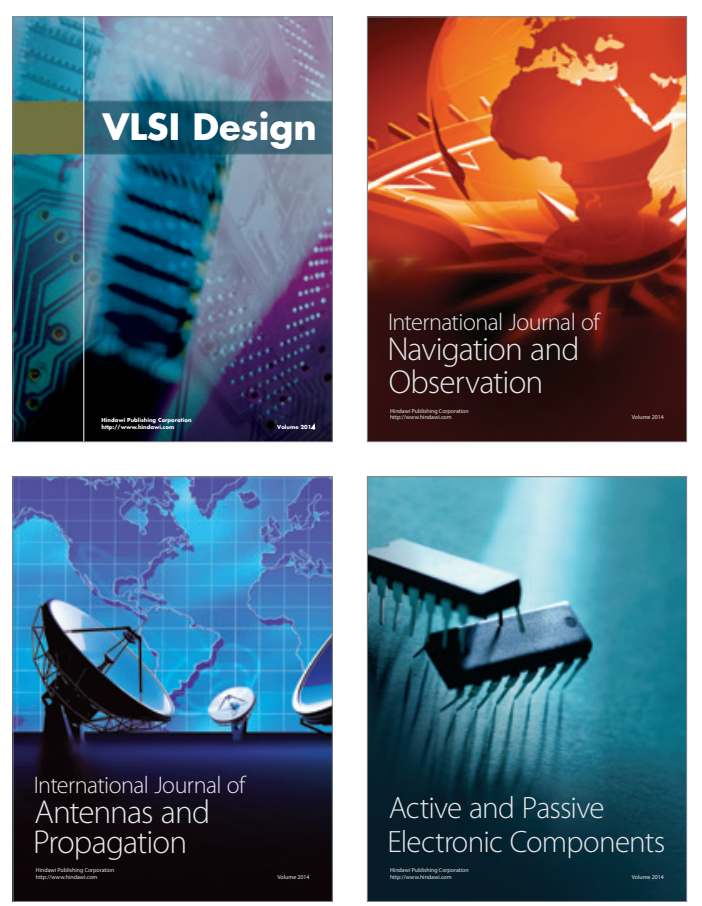
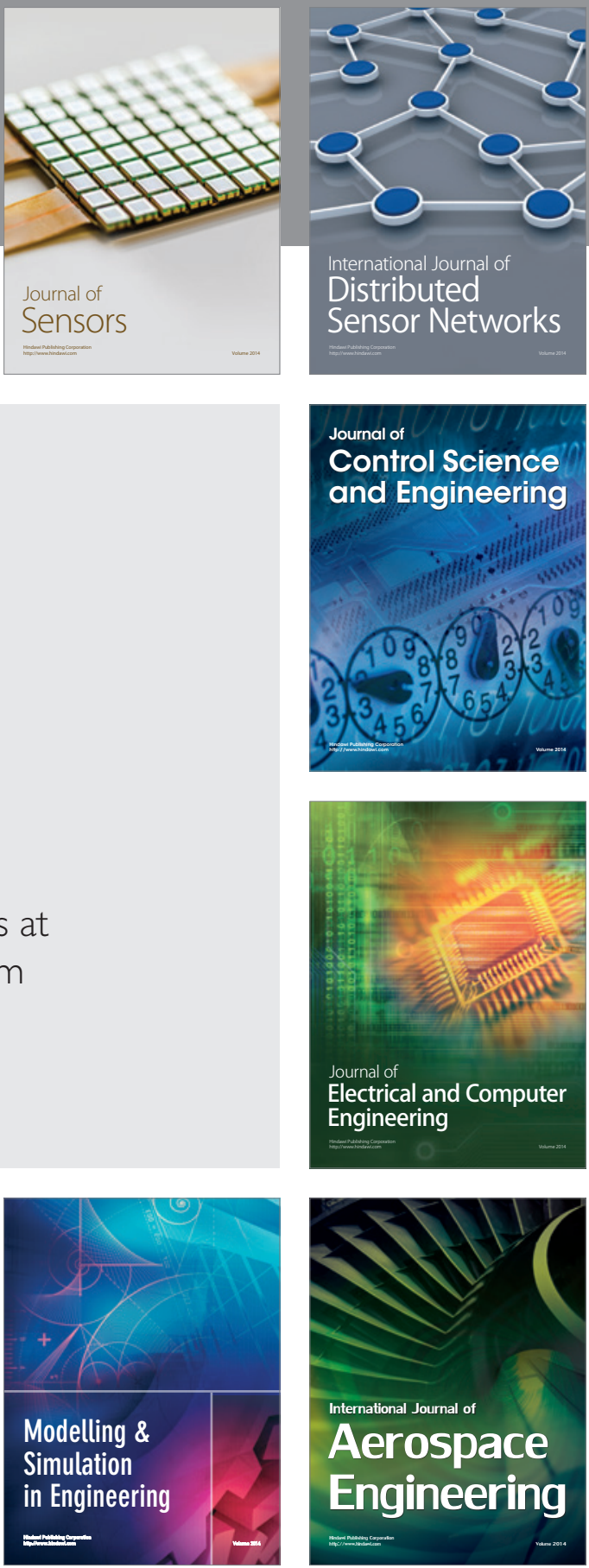

Journal of

Control Science

and Engineering
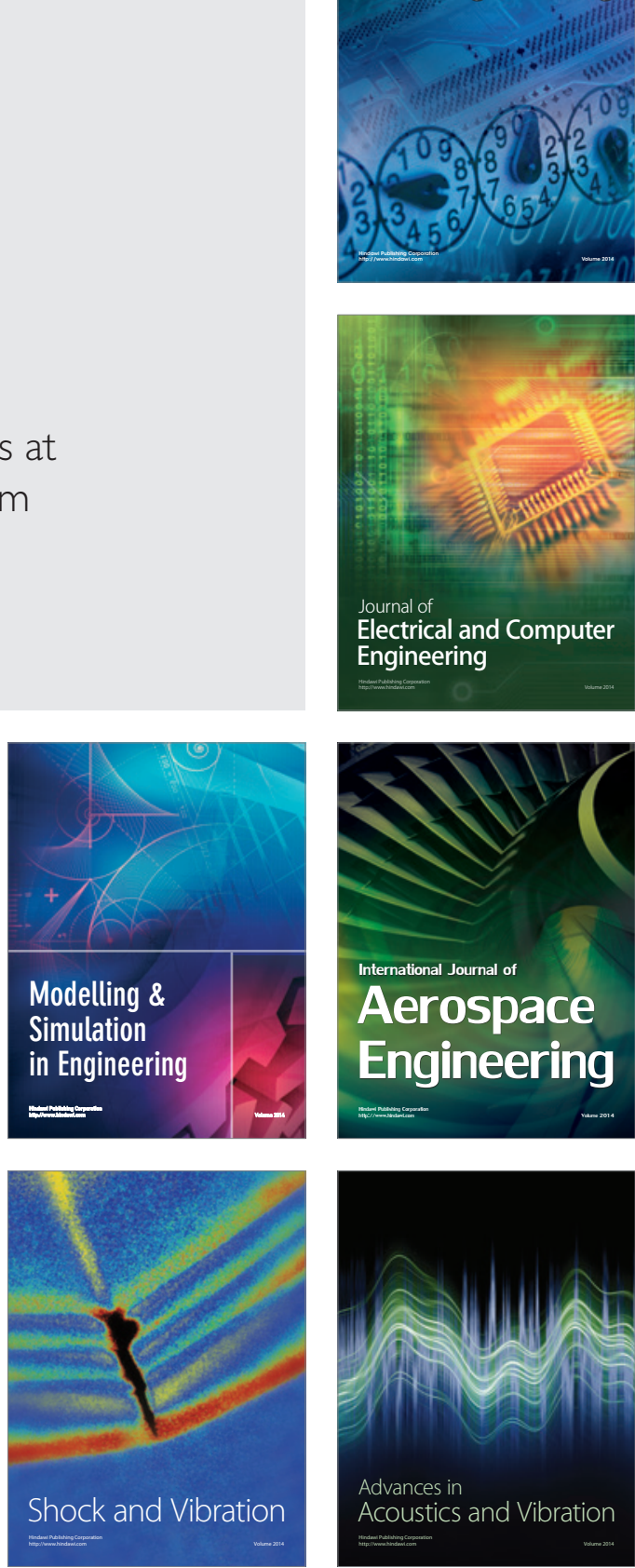